\title{
Post-Traumatic Stress Disorder \& Treatment Approaches Adult Psychopathology
}

\author{
Dr. Phillip D. Clingan
}

\begin{abstract}
The research paper addresses the problem of a psychological disorder called post-traumatic stress disorder (PTSD), and compare and examine treatment approaches, (1) stress inoculation, and treatment approach, (2) exposure therapy. A solution to this problem will help combat veterans and people who have PTSD, to include mental health professionals experiencing difficulties managing PTSD patients who express anger, anxiety, depression, fear memories and feelings of helplessness during therapeutic sessions to help control negative influences. To solve this problem, the researcher identifies through a research paper, peer-review journals, primary and secondary resources. These resources examine through using describing the psychological disorder, examining the effectiveness of two treatment approaches, comparing the treatment approaches, and analysis can recognize and decrease PTSD symptoms. Consolidated, the resources introduce primary treatment strategies such as psychotherapy, medications, treatment approaches that include stress inoculation, exposure therapy, self-care mindfulness, individual and group therapy, and structured approach therapy frameworks. This approach compares and summarizes sources used to address the research paper, comparison of treatment approaches, and emotional responses of veteran participants. The approach continues to examine the impact of client treatments, feedback from PTSD patients regarding group-based exposure therapy, and the need for further research. The research paper addresses how PTSD can make a veteran feel like the traumatic event is happening over-and-over, and how group-based exposure therapy (GBET) offers advance mental health care for survivors of traumatic events.
\end{abstract}

Index Terms - Post Traumatic Stress Disorder; Group-based Exposure Therapy; Depression.

\section{INTRODUCTION}

Post-traumatic stress disorder (PTSD) may be a simple concept about a traumatic event, and the definition is usually different, but PTSD is a powerful life experience that may have life-long effects. About $5 \%$ of men and $10 \%$ of women become diagnosed with PTSD in their lifetime ("PTSD," 2014). That is 1 in 30 adults in the United States in any year suffer from PTSD, and that number is increasingly higher in combat veterans. More important, that PTSD exists beyond war, and any event that threatens and leaves one feeling helpless, horror, or fear ("PTSD," 2014).

Even though PTSD can make one feel threatened and unsafe or makes one feel helpless. When an individual experience a traumatic event, it is reasonable to feel anxious, sad, and disconnected. If the pain does not leave, and we feel a constant sense of danger and hurtful memories, we may have PTSD., it can seem like we never feel normal again or recover from what has happened, by reaching out and seeking treatment, support, and developing new coping skills, we can move forward in one's life and overcome PTSD.

\section{1) Purpose Statement}

The goal of this research paper is to describe and examine different treatment approaches for post-traumatic stress disorder (PTSD), describe PTSD, discuss and compare treatment approach (1) stress inoculation, and (2) exposure therapy. The following summarizes the sources used, as supportive evidence that group-based exposure therapy significantly reduces psychiatric symptoms and depression. Studies reveal that in combat veteran's depression mediates the relationship between learning, PTSD, and memory impairment. That GBET builds group cohesion and increases the likelihood that veterans will support one another by providing an element that provides an opportunity for nurturance and socialization.

\section{METHOD}

\section{1) Overview of Post-Traumatic Stress Disorder}

Every individual face danger and fear differently, and when people feel they are in danger, it is human nature to feel afraid. Fear stimulates many changes within the body to prepare to avoid the threat or defend against it. The "fight-or-flight" reaction is a normal response designed to protect an individual from harm. However, in PTSD, these reactions described above become damaged or changed. Individuals with PTSD encounter feelings of fear or stress though the danger is no longer present ("PTSD," 2014).

The development of PTSD usually occurs following a horrific ordeal that threatens a person with physical harm or involved physical damage. This person develops PTSD once they become directly harmed or if harm affected a family member, loved one or the individual witnessed the traumatic event that happened to strangers or their loved ones. The public associates typically PTSD with war veterans, but any person can experience traumatic events such as mugging, raped, child abuse, torture, kidnapped, car wrecks, train wrecks, bombings, natural disasters, earthquakes and floods which can cause the person developing PTSD ("PTSD," 2014). 


\section{2) Causes and Brain Areas}

Genetically, many researchers are focusing on if genes play a role in creating memories of fear. Imagine if a scientist found how fear memories exist. Not only would society understand PTSD, but might refine interventions for reducing symptoms of PTSD. For example, mice create a protein called stathmin needed to form fear memories. If a mouse does not produce this protein, then the mouse is less likely to exhibit protective responses to fear or danger when exposed to a traumatic event Gastrin-releasing peptide (GRP), a brain-signaling chemical released when a person experiences an emotional affair. Imagine if GRP in mice controls their fear response and mice that do not produce GRP experiences longer-lasting memories of fear ("PTSD," 2014).

Recently, researchers have discovered a variant of the serotonin-transporter-linked polymorphic region, (5-HTTLPR) gene, which controls a brain chemical called serotonin related to moods that feed fear responses. Comparably, many other mental disorders, PTSD may have many genes working at once to impact the PTSD symptoms. By studying the human brain, a scientist can understand how the mind becomes involved with stress and fear, which may discover probable causes of PTSD. One specific area of the brain structure, specifically of interest is the amygdala for its role in memory, learning, and emotion. This area of the brain remains known for learning fear, such as a fear acquisition. For example, your brain learns fear through an event such as touching a hot stove ("PTSD," 2014).

The area of the brain that stores both the original fear responses and extinction of memories called the prefrontal cortex (PFC). The PFC also performs a task such as problem-solving, decision-making, and judgment. Like any other body part, the PFC plays different roles within itself. For example, the PFC suppresses controllable stress in the amygdala (the alarm center in the brainstem), which controls the responses of anxiety. The section of the PFC called ventromedial sustains long-term fearful memories, and more importantly, the brain size of this area may affect the PFC's ability to store long-term extinction of fear memories ("PTSD," 2014).

\section{3) Statement of Main Points}

These distinctive differences in these brain areas or genes may help put a spotlight on PTSD without causing symptoms. Early growth of the brain may be at risk when a person experiences environmental factors such as head injury, childhood trauma, or mental illness. Also, cognitive, personality and social factors such as tendencies to view life challenges negatively or positively influence how individuals adjust to trauma. Perhaps one-day scientist can use all or a combination of these factors to predict what individuals will develop PTSD following a traumatic event ("PTSD," 2014).

\section{4) Sign and Symptoms}

There are three categories of PTSD symptoms: first, re-experiencing symptoms, such as bad dreams, flashbacks where the patient is reliving the trauma repeatedly, this may include sweating and elevated heart rate, and frightening thoughts. Second, avoidance symptoms that may consist of feeling intense guilt worry, depression, feeling emotionally numb, and difficulty remembering dangerous events. And staying away from games that remind the individual of a lousy experience and losing interest in activities once enjoyed. Last, hyperarousal symptoms such as feeling edgy, tense, feeling easily startled, having anger outburst, and difficulty sleeping ("PTSD," 2014).

\section{5) Diagnosis}

A traumatized person may never develop minor or full-blown PTSD. Symptoms described above usually begin within two months but can emerge years later following the traumatic event. Most importantly, the symptoms have to persist more than a consecutive month to be considered PTSD. The course of PTSD varies with every person, where some recover immediately, and other people experience chronic symptoms. A person must undergo the following for at least one month before receiving PTSD diagnoses: a) at least two hyperarousal symptoms, b) one re-experiencing symptom, and c) three avoidance symptoms. And a PTSD individual may suffer from substance abuse, depression, and more than one anxiety disorder ("PTSD," 2014).

\section{6) Who Is at Risk}

Everyone. Anyone at any age can get PTSD, and this illness affects over 7.7 million Americans, but more importantly, this illness can occur at any age. To reinforce what anyone means, this includes individuals who have experienced abuse, sexual assault, disasters, accidents, and many other traumatic events. Statistically, women are more likely than men to develop PTSD, and recent research reveals PTSD may be hereditary. What more, just because a person experiences a traumatic event does not mean they will experience PTSD symptoms? Many people get PTSD after a family member or friend dies unexpectedly ("PTSD," 2014).

\section{7) Why People Have PTSD and Why Others Do Not}

One crucial fact to remember is that not all people who experience a traumatic event will get PTSD. Many factors determine if a person receives PTSD or is resilient to the risk of PTSD. Understanding that risk and resilient factors are present before, during, and after a traumatic event helps the individual understand the overall impact of PTSD factors. Risk factors include getting hurt, the family history of mental illness, feeling helpless, extreme fear, dealing with extra stress, loss of a job, a loved one, or home. Resilience factors may reduce risk factors of PTSD include having a coping strategy, establishing methods to deal with stress, loss, and learn from the event. Also, seeking the support of the community, people, family, and friends. Identifying social support groups related to the specific traumatic event, and dealing with an event we live through ("PTSD," 2014). 


\section{8) Treatments}

The primary treatment approach for individuals who have PTSD is medications, psychotherapy, or both. Special note should be made that every person is different, so what works for one patient may not work for another PTSD patient. More important, the PTSD patient should be treated by a mental health care professional or a health care provider experienced with PTSD patients. And due to people differing from one another, this includes different approaches must be taken for each person. For example, one PTSD patient may be in an abusive relationship so both physical, emotional, panic disorder, substance abuse, depression, and other disorders and behaviors will be addressed ("PTSD," 2014).

\section{9) Treatment Approach: Stress Inoculation}

The primary focus using stress inoculation therapy is to reduce PTSD symptoms through anxiety reduction and teach the patient to see their memories as a healthy and positive manner ("PTSD," 2014). Family stress directly contributes to poor PTSD outcomes, so one way to reduce family stress and increase support is to combine couple and family therapy to reduce PTSD symptoms [5].Structured approach therapy (SAT) is a theoretical framework that operates within the stress inoculation framework. Therefore, the stress inoculation develops skills to help cope not only with PTSD but buffer against future stressful events [5].

The training stress inoculation provides within the framework of SAT works for several reasons. First, stress inoculation provides couples with skills they can use in their relationship, traumatic stress, and future relationship stressors. Second, integrating treatment procedures and training into one framework. Third, stress inoculation research has efficacious in psychological problems such as anger management, PTSD, and phobic behavior. Last, stress inoculation can be tailored to meet individual and couple's needs. What's more, stress inoculation consists of three phases: a skills training phase, the educational phase, and the application phase [5].

During the education phase, the patient and couple focus on building a therapeutic relationship, view stress as transactional and conceptualize presenting stressors or problems. The skills training educates coping skills then slowly integrate coping strategies into the couple's relationship. Finally, the application-phase encourages couples to apply all that they have learned throughout the SAT framework in the present and future. More critical, SAT considers emotional numbing and avoidance as not just PTSD symptoms, but mechanisms that hinder PTSD recovery from traumatic events [5].

Another approach to stress inoculation training is the self-care mindfulness approach for PTSD individuals. Self-care mindfulness is relatively new, with little research history but seems to expand rapidly within the Veteran Administrations (VA) clinical practices for PTSD [1].One important note when comparing PTSD strategies is that self-care mindfulness is unused as a first-line treatment for PTSD. The mindfulness-based approach facilitates the PTSD patient's hyperarousal symptoms and comorbid conditions.
Unlike SAT, mindfulness-based approach has a history of treating a variety of psychiatric, psychological, and physical problems, including depression, PTSD, chronic pain, and anxiety [1].

Self-care helps PTSD individuals manage their illness and increase levels of functioning, decreased health care costs, improved health outcomes, and reduced pain. Self-care interventions have become useful in reducing the frequency of intrusive symptoms, avoidance behavior, anxiety, increase coping skills, and decrease depressive symptoms. More critical, self-care mindfulness approaches are portable, safe, easy to learn, affordable, and due to the increasing effectiveness of the approach, may help optimized PTSD treatment standards. What's more, mindfulness programs support President Obama's August 31, 2012, Executive Order in developing strategies that improve the prevention and treatment of PTSD [1]. Mindfulness is the ability to maintain non-judgmental awareness, openness, acceptance, and support one's self moment-by-moment. There are four characteristics of mindfulness, openness, curiosity, love, and acceptance. Mindfulness impacts psychological functioning and improves overall health. Modern, group-based meditations such as mindfulness-based cognitive therapy (MBCT) include yoga and tai chi, and also include relaxation training within psychological interventions relevant for PTSD [1].

Mindfulness enhances focused attention by supporting the lateral prefrontal cortex (PFC) and the anterior cingulate cortex. Unlike SAT, mindfulness improves emotional management through the PFC inhibition of the amygdala, which advances the stability of non-conceptual sensory pathway [1]. Therefore, mindfulness controls negative emotions by becoming an alternant to cognitive efforts. A recent study shows mindfulness-based stress reduction may influence the hypothalamic-pituitary-adrenocortical (HPA) axis adjusting the cortisol levels [1].

Unlike the SAT model, the interventions of mindfulness use relaxation techniques that target the autonomic nervous system, lower blood pressure, help to breathe, and promote well-being and calm feelings. Comparatively, recent PTSD studies show mindfulness relaxation training desensitized the following PTSD-related symptoms: numbing and hyperarousal, attrition, reductions in guilt, and anger. Supportive evidence exists for MBCT for prevalent PTSD comorbidities, including anxiety, depression, stress reduction, and chronic pain [1].

\section{0) Treatment Approach: Exposure Therapy}

Specifically intended to help PTSD individuals control and face their fear. The therapist will expose the patient to the trauma they experienced positively. The process includes visiting the location where the trauma took place, writing, or mental imagery. These tools help mental health professionals help PTSD patients cope with their feelings ("PTSD," 2014). The

VA health care system uses over 140 PTSD specialized treatment programs specifically for veterans suffering from severe and chronic PTSD. Group-based exposure therapy 
(GBET) is a multifaceted, manualized, and cognitive-behavioral treatment approach since 2003. Primarily, GBET synthesizes components of psychosocial skills training, psychological education to treat chronic trauma memories of combat-related PTSD, and depressive symptoms [4].

Unlike SAT and mindfulness eight-week training model, GBET veterans may meet twice a week, three hours a day, for up to 16 weeks. In a recent study (Ready et al., 2012), 30 veteran PTSD participants gave an in-group combat-related story, then listen to the recording ten times. During the follow-up phase, PTSD symptoms reported during the pretreatment assessments were lower when collected between 7 to 11 months. The study reported a mild recording of depression. Establishing and using a Beck Depression Inventory 2 (BDI-2), and the PTSD checklist (PCL) administered during pretreatment and post-treatment assessments [4].

The GBET method varies in one particular aspect to SAT and MBCT therapy, which GBET requires group members to call one another between sessions during the first two phases. Other forms of treatment mentioned are research evaluators making independent calls to the participants where GBET encourages participants to stay in touch during and after the group sessions. Exposure therapy requires participants to present two half-hour speeches about their lives before combat, all while applying grounding techniques and coping skills. This process is goal-oriented to create a feeling of trust and safety among the veterans within the group [4].

Opinions can be understood that combat veterans sitting in a group session listening to traumatic events may be harmful, but during this study of 30 veterans, no evidence occurred in GBET. Many reasons for facilitating GBET include veterans revealing intimate details and hearing validations from other veterans within a group setting with similar traumatic experiences. These shared experiences may normalize the adverse responses they had in combat. Members of the group may identify with symptoms and dysfunctional behaviors following trauma, including depression behaviors. Referring to these promising benefits, others are augmenting extended GBET model settings [4].

A different approach to understanding GBET stems from a recent study (Mott et al., 2013), of receiving 20 combat veterans' perspectives of self-reporting GBET experiences. The concern that supports this approach is due to the substantial debate regarding GBET settings. Some researchers and clinicians argue that group therapy vicariously traumatized patients while the other group settings may expose opportunities for social support and normalization. There are very few studies of combat veterans and what their opinions are of treatment and group exposure. These opinions are veteran's perspectives of a 12-week GBET for PTSD [2].

Of 20 veterans providing GBET feedback, 18 completed the survey. Initially, $78 \%$ disclose they considered dropping out of treatment, while $28 \%$ contemplated leaving GBET during the first phase. Just $6 \%$ admitted to strong desires of dropping out daily, and $17 \%$ did not indicate leaving during all phases of GBET. When asked to justify their responses, all members expressed a commitment to the group. One veteran stated, "I did not run out on my men in Vietnam, and I was not going to do it here." When the veterans expressed the potential benefits of GBET, a veteran stated, "I wanted to see how this would help me," while another said, "I had agreed to do this, and would not back out." The feedback supported the power of unity and that veterans were not only committed to themselves, or the GBET group but also in showing dedication and honesty staff members [2].

\section{RESULTS}

\section{1) Summary}

Overall, self-care mindfulness training decreased avoidance behaviors when compared to the SAT framework, by improving impulse control in prevention of comorbid substance use, aggression, and emotional regulation. Both SAT and mindfulness help reduce ruminating negative thoughts, but mindfulness demonstrated results in decreasing depression relapse and PTSD symptoms. Comparatively, GBET suggest that Vietnam veterans and recent combat veterans present with chronic PTSD symptoms and depression greatly benefit from exposure-based therapy. More critical, GBET reduces psychiatric symptoms when exposure therapy treatments become systematically applied.

\section{2) Analysis}

Research conducted using behavioral family therapy (BFT) with PTSD veterans and one family member assigned to a control condition list that involved exposure therapy or exposure therapy with BFT. Treatment involving BFT showed little reductions in PTSD symptoms when compared to treatment without family intervention, but there were small advances in those who received BFT [5]. The SAT model requires couples to attend twelve to fifteen, 60-minute sessions and has only been studied with combat-related PTSD, not PTSD that stems from other traumatic events.

A recent VA survey of 125 PTSD veterans indicated that $88 \%$ of their treatment program offered self-care mindfulness practices such as meditation and yoga. The mindfulness approach requires an 8-week training course and a six-month follow-up. Mindfulness training mitigates PTSD symptoms both immediately regarding post-treatment and at follow-up three months later. During the GBET research study of PTSD and depression, of 30 veterans, $73 \%$ reported reductions on the BDI-2 assessment or PCL checklist. 36\% self-reported they no longer meet PTSD criteria during the PCL follow-up assessment [4].

\section{DISCUSSION}

\section{1) Need for Further Research}

In conclusion, the results to describe and examine two treatment approaches for the psychological disorder of post-traumatic stress disorder (PTSD), describes PTSD, discuss and compare treatment approach (1) stress vaccination, and treatment approach (2) exposure therapy. The research paper illustrated two successful psychotherapeutic PTSD approaches to reduce anger, anxiety, 
depression, and feelings of helplessness. Evaluating and comparing peer journals of PTSD treatment approaches support a validation that GBET helps PTSD individuals face traumatic events.

Overall, the research paper achieved its goal illustrating the importance of PTSD treatment strategies, frameworks, veteran GBET critiques, and the importance of group cohesion during treatment sessions. More important, those positive results much depend upon the experience of therapists, the treatment approach, and personal history of the individual. More research remains needed to understand the importance of recognizing PTSD symptoms before, during, and after a therapeutic session to help reduce distress, helplessness, and depression so people will feel safe and secure.

\section{ACKNOWLEDGMENT}

I am using this opportunity to express my gratitude to my family and friends who supported me throughout the course of this research project. I am thankful for their aspiring guidance, invaluably constructive criticism and friendly advice during the project work. I am sincerely grateful to them for sharing their truthful and illuminating views on a number of issues related to the project.

\section{REFERENCES}

[1] Khusid, M. (2013). Self-Care Mindfulness Approaches for Refractory Posttraumatic Stress Disorder. Psychiatric Annals, 47(7),340-344.http://dx.doi.org/10.3928/00485713-20130703-11

[2] Mott, J. M., Sutherland, R. J., Williams, W., Lanier, S. H., Ready, D. J., \& Teng, E. J. (2013). Patient Perspectives on the Effectiveness and Tolerability of Group-Based Exposure Therapy for Posttraumatic Stress Disorder: Preliminary Self-Report Findings From 20 Veterans. Psychological Trauma: Theory, Research, Practice, and Policy, 5(5), 453-461. http://dx.doi.org/10.1037/a0029386

[3] Post-Traumatic Stress Disorder (PTSD). (2014). Retrieved March 25, 2014, from http://www.nimh.nih.gov/health/topics/post-traumaticstress-disorder-ptsd/index.shtml\#part1

[4] Ready, D. J., Sylvers, P., Worley, V., Butt, J., Mascaro, N., \& Bradley, B. (2012). The Impact of Group-Based Exposure Therapy on the PTSD and Depression of 30 Combat Veterans. Psychological Trauma: Theory, Research, Practice, and Policy, 4(1), 84-93. http://dx.doi.org/10.1037/a0021997

[5] Sautter, F. J., Armelie, A. P., Glynn, S. M., \&Wielt, D. (2011). Developing a Couple-Based Treatment for PTSD inReturning Veterans. Professional Psychology: Research and Practice, 42(1), 63-69. http://dx.doi.org/10.1037/a0022323

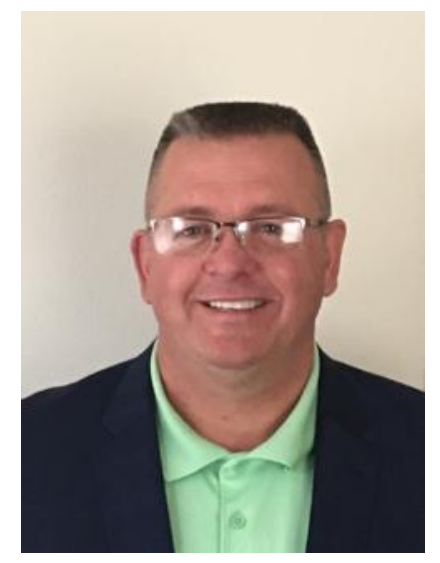

Dr. Clingan after serving 21 years in the United States Army undertook his Psy.D in the Department of Psychology, specializing in criminology in the School of Organizational Leadership at the Ashford University, Denver, Colorado. He is a faculty member teaching in the Departments of Psychology and Criminal Justice in San Antonio, Texas for Pierce College and serves as a Content Expert and Dissertation Chair for Grand Canyon University School of Doctoral Studies regarding quantitative and qualitative studies for doctoral candidates. Dr. Clingan specializes in preeminent psychology as applied within the field of criminology. His psychology work is based upon field experience in criminal trials since 2013 and heavily influenced by the theoretical frameworks of Dr. Paul Ekman and measuring nonverbal communication. Loves writing and riding his Indian Motorcycle. 